\section{L'Actualité économique}

L'ACTUALITÉ ÉCONOMIQUE

\section{A. Asimakopulos et la Théorie générale de Keynes}

\section{Gilles Dostaler}

Volume 67, numéro 4, décembre 1991

URI : https://id.erudit.org/iderudit/602054ar

DOI : https://doi.org/10.7202/602054ar

Aller au sommaire du numéro

Éditeur(s)

HEC Montréal

ISSN

0001-771X (imprimé)

1710-3991 (numérique)

Découvrir la revue

Citer cet article

Dostaler, G. (1991). A. Asimakopulos et la Théorie générale de Keynes. L'Actualité économique, 67(4), 549-566. https://doi.org/10.7202/602054ar
Ce document est protégé par la loi sur le droit d'auteur. L'utilisation des services d'Érudit (y compris la reproduction) est assujettie à sa politique d'utilisation que vous pouvez consulter en ligne.

https://apropos.erudit.org/fr/usagers/politique-dutilisation/ 


\title{
A. Asimakopulos et la Théorie générale de Keynes*
}

\author{
Gilles DOSTALER \\ Département de sciences économiques \\ Université du Québec à Montréal
}

\section{INTRODUCTION}

Athanasios (Tom) Asimakopulos s'est éteint à Montréal le 25 mai 1990, dans sa soixantième année, des suites d'une forme rare de leucémie contre laquelle il a lutté avec un courage exemplaire durant les deux dernières années de sa vie ${ }^{1}$. Jusqu'à la fin, il n'a cessé d'écrire, achevant peu avant sa mort un livre qui couronne son apport exceptionnel à la compréhension de la pensée de John Maynard Keynes, Keynes's General Theory and Accumulation (Asimakopulos, 1991). Un flot ininterrompu d'articles de revue et de contributions à des ouvrages collectifs continuent de paraître: plus d'une douzaine de titres depuis 1989.

L'analyse critique de la Théorie générale de Keynes constitue un thème important dans l'œuvre de Tom Asimakopulos. Ses nombreux articles consacrés à ce grand économiste sont le fruit d'un effort d'explication dont les premiers destinataires étaient ses étudiants. L'approche d'Asimakopulos à l'œuvre de Keynes est originale et stimulante. Elle se démarque de ce qu'on appelle, à la suite de Paul A. Samuelson, la synthèse néo-classique, sans pour autant en rejeter tous les éléments. Asimakopulos a d'ailleurs bien mis en lumière les positions nuancées, et parfois fluctuantes, sur l'interprétation à donner à la Théorie générale, qu'on peut trouver chez des auteurs comme John Hicks, Harry Johnson ou Don Patinkin. Sa propre lecture est plus proche de celle du courant qualifié de post-keynésien, mais l'une des caractéristiques de sa démarche intellectuelle a été de ne jamais adhérer de manière dogmatique à une école. Ce qu'on appelle le courant postkeynésien est d'ailleurs une entité complexe, difficile à cerner et, tout comme ce qu'on appelle la théorie néo-classique, très diversifiée. Il comporte de nombreux sous-courants et vit d'intenses querelles intestines. Il y a aussi des recoupements

* Une première version de ce texte a été présentée comme communication au $31^{\circ}$ congrès annuel de la Société canadienne de science économique, à l'Université Laval, de Québec, le 16 mai 1991. Nous sommes reconnaissant à Marika Asimakopulos pour avoir mis à notre disposition les épreuves du livre de son époux. Nous remercions Claire de la Durantaye pour ses commentaires sur notre texte.

1. Pour un aperçu de la carrière de Tom Asimakopulos, voir Hamouda (1990) et Harcourt (1991). Nous avons eu l'occasion d'apprécier les qualités pédagogiques exceptionnelles et la disponibilité de Tom pendant nos études à l'Université McGill, entre 1968 et 1971. Depuis, il était devenu un ami et un collègue. 
entre post-keynésiens et néo-classiques. Ce sont à des auteurs, plutôt qu'à des écoles, qu'Asimakopulos associe sa démarche. Deux d'entre eux ont joué un rôle important dans l'évolution de sa pensée, Michal Kalecki et Joan Robinson². Mais, pas plus que de Keynes, il ne fut un disciple inconditionnel de l'une ou de l'autre. Il a bien souligné, dans certains de ses textes les plus récemment publiés, la nature complexe des relations intellectuelles entre Keynes, Robinson et Kalecki (1988d, 1989c, 1990a). C'est d'ailleurs ce dernier, plus que Keynes, qui a formé la vision de Robinson, et qui est de ce fait le véritable inspirateur de ce qu'on appelle l'école post-keynésienne ${ }^{3}$. Il est utile de rappeler quelques éléments de l'itinéraire de Kalecki, compte tenu de l'importance des références d'Asimakopulos à cet auteur.

Né en Pologne, autodidacte en théorie économique Michal Kalecki a publié en 1933, dans son pays et sa langue d'origine, une analyse des fluctuations cycliques qui contient certaines des thèses de la Théorie générale de Keynes. Le débat sur le fait de savoir à quel point la théorie keynésienne a été anticipée par Kalecki est loin d'être clos. Lawrence Klein, Joan Robinson ou George Shackle voient en lui un précurseur à certains égards supérieur à Keynes. Pour sa part, Don Patinkin nie qu'on puisse considérer les travaux de Kalecki comme des anticipations de la Théorie générale (Patinkin, 1982). Asimakopulos admet en partie la conclusion de Patinkin (1983a). Alors que Keynes voulait expliquer la possibilité d'un équilibre de sous-emploi en courte période, Kalecki cherchait à construire un modèle endogène des fluctuations cycliques de l'économie. Inspiré par Marx et sa compatriote Rosa Luxembourg, Kalecki considérait que le chômage était une situation normale dans une économie capitaliste. Keynes cherchait de son côté à se libérer d'une théorie orthodoxe, qu'il appelait «classique», incapable d'expliquer le chômage involontaire. Toutefois, les éléments essentiels des cadres théoriques de Kalecki et de Keynes, en particulier la nature des liens entre investissement, épargne, production et emploi, sont analogues. De sorte qu'il aurait été possible, à partir du modèle proposé par Kalecki en 1933, de développer la problématique de la Théorie générale (Asimakopulos, 1983a). Kalecki le croyait sans doute lui-même. Il se trouvait à Stockholm en 1936, et il a lu la Théorie générale alors qu'il s'apprêtait à écrire un livre analogue. Il s'est alors rendu à Cambridge, après un passage par la London School of Economics. Dès ce moment a commencé une collaboration étroite avec Joan Robinson, qui a été très impressionnée par l'économiste polonais (Asimakopulos, 1988d et 1989c). L'accueil de Keynes a été plus froid. Dans un de ces derniers articles parus (1990a), Asimakopulos a analysé les points d'accord et de désaccord entre les deux auteurs à partir d'un examen de la correspondance reliée à la soumission d'articles par Kalecki à l'Economic Journal. Keynes a d'ailleurs refusé l'un de ses articles. Mais il s'est aussi rallié, dans des articles postérieurs

2. "I have accumulated many intellectual debts in arriving at my interpretation of Keynes, and in the writing of this book. Robinson's writing on the General Theory and on Kalecki started me off on my present road, and the strong impression they had on me was confirmed by personal contacts with her from 1970" (Asimakopulos, 1991, p. xvi-xvii).

3. Voir à ce sujet Dostaler (1988). 
à la publication de la Théorie générale, à certaines des thèses de Kalecki ${ }^{4}$. Ce dernier, quant à lui, n'a fait qu'une brève allusion, en 1942, au fait qu'il avait développé indépendamment de Keynes certaines des thèses de la Théorie générale. Il n'a été plus explicite à cet égard que dans l'introduction à un recueil de ses principaux articles, publié à titre posthume en 1971, y soulignant que ses premiers articles en polonais contenaient l'essentiel de la Théorie générale de Keynes.

\section{LA THÉORIE GÉNÉRALE ET LA THÉORIE GÉNÉRALE}

C'est à l'œuvre de Keynes qu'est consacré le dernier livre de Tom Asimakopulos, et non pas à celle de ses disciples, exégètes, ou prédécesseurs, si l'on fait abstraction des deux derniers chapitres contenant un examen critique des modèles d'accumulation de Roy Harrod et Joan Robinson. Plus précisément, il s'agit de reconstruire une théorie, dérivée de la Théorie générale, mais aussi de textes ultérieurs de son auteur. Car Keynes est lui-même intervenu, durant les dix années qui lui restaient à vivre après la publication de son livre, dans la querelle sur son propre héritage. Il a transmis des signaux ambigus et parfois contradictoires sur le sens de son œuvre, approuvant par exemple le «Mr. Keynes and the Classics» de Hicks tout en développant, dans le Quarterly Journal of Economics de février 1937 (CW, vol. 14, 109-23), des thèses en totale contradiction avec ce qui allait devenir le modèle IS-LM. Asimakopulos croit néanmoins qu'on peut retrouver une cohérence dans les interventions de Keynes: «Il y a donc place pour un examen attentif de la Théorie générale de Keynes qui se base sur ses écrits, mais en corrige aussi les erreurs théoriques mineures, et traite des ambiguités qu'on trouve dans l'exposé, par Keynes, de sa théorie. L'élaboration d'une telle présentation constitue l'un des deux buts majeurs de cet ouvrage» (Asimakopulos, 1991, p. 3) . On $^{5}$ remarquera que l'expression de «Théorie générale», dans la citation qui précède, n'est pas en italiques comme on le ferait pour le titre d'un livre. Il en est de même pour le titre du livre d'Asimakopulos. Son auteur prend bien soin, en effet, de distinguer le livre de Keynes d'une théorie qu'on y trouve partiellement élaborée, mais qui ne prend tout son sens qu'à la lumière d'écrits ultérieurs de Keynes. Joan Robinson avait déjà proposé une telle distinction, en 1948, alors que commençait à s'imposer cette synthèse néo-classique qu'elle considérait comme un keynésianisme abâtardi, dont Keynes se serait parfois, à son avis, lui-même fait le complice:

«Mais par théorie générale je ne désigne pas le livre célèbre de cet auteur. Certes, cet ouvrage est très important, mais il n'est ni complet ni définitif. Il constituait, lors de sa parution, une sorte de compte rendu provisoire sur un mouvement d'idées en cours de développement. [...]

4. On trouvera ces articles dans le vol. 14 des Collected Writings of John Maynard Keynes, que nous désignerons désormais par $\mathrm{CW}$, suivi du numéro du volume.

5. Notre traduction, comme pour toutes les autres citations tirées de publications anglaises. Nous laisserons les citations reproduites dans les notes dans la langue originale. Voici comment Asimakopulos présente le deuxième objectif de son livre: «The second aim of the present book is to examine the attempts by two of Keynes's younger associates, R. F. Harrod and Joan Robinson, to start from Keynes's analysis and to develop theories that deal with the growth of an economy over time» (Asimakopulos, 1991, p. 9). 
Ce que j'entends par l'expression de théorie générale est plutôt une méthode d'analyse. C'est un corps vivant d'idées qui se développe et qui produit des résultats très différents suivant qu'il est appliqué à des circonstances différentes par telle ou telle personne.» (Robinson, 1948, p. 185)

Le livre de Keynes, par opposition à la Théorie générale ainsi définie, se caractérise par le fait qu'on y trouve imbriquées plus d'une méthode d'analyse, et qu'elles ne sont pas nécessairement cohérentes. C'est l'ouvrage d'un homme qui, de son propre aveu, avait cherché péniblement à se libérer d'un certain nombre d'idées reçues, d'une orthodoxie dans laquelle il avait reçu son éducation économique. Des traces de cette orthodoxie demeurent donc présentes dans l'ouvrage. C'est de l'intérieur que Keynes a cherché à détruire la citadelle de ce qu'il a appelé la théorie classique. Des morceaux de l'ancien sont utilisés pour construire le nouveau. Si l'on ajoute à cela le fait que la Théorie générale a été publiée dans une certaine hâte, nonobstant son long temps de gestation, compte tenu de l'urgence politique ressentie par Keynes ${ }^{6}$, il n'est pas étonnant qu'elle puisse donner lieu à tant d'interprétations contradictoires ${ }^{7}$.

La Théorie générale contient donc «quelques erreurs théoriques mineures ainsi que des formulations apparemment contradictoires à propos d'éléments-clés de la théorie, tels que la détermination de l'investissement» (ibid., p. 2). Elles ont en partie leur source dans la méthode de travail de Keynes, dans son approche de la réalité économique. Parmi les qualités qui font la force des travaux de Keynes, on compte son intuition et sa connaissance profonde du fonctionnement et des institutions du capitalisme contemporain. Sa vision théorique se fonde sur une connaissance concrète, pratique, de la société dans laquelle il vit. Son objectif ultime est de convaincre ses contemporains de l'urgente nécessité d'opérer des transformations profondes pour éviter un écroulement des sociétés modernes, gangrenées par des taux de chômage inacceptables et une répartition trop inégalitaire des revenus. Keynes a lui-même expliqué que le bon économiste doit réunir en lui des qualités qu'on trouve rarement chez un seul homme, parmi lesquelles une bonne connaissance tant de l'histoire que de la philosophie, une intuition sûre, des qualités d'homme d'État, et la capacité de construire des modèles.

Keynes ne valorisait pas pour elles-mêmes la perfection formelle et l'élégance des modèles théoriques. On sait aussi que, durant ses années de formation univer-

6. La même situation avait prévalu au moment de la publication, en 1930, du Treatise on Money (CW, vol. 5 et 6). Dans sa longue critique de ce livre, Hayek a mis de l'avant cette hypothèse pour expliquer les nombreuses incohérences théoriques qu'il croyait y déceler. Keynes s'est d'ailleurs très vite déclaré insatisfait de son œuvre, et en a entrepris une révision qui a abouti à la Théorie générale. C'est par crainte d'une nouvelle opération de ce genre que Hayek s'est abstenu de critiquer la Théorie générale, ce qu'il a regretté par la suite. De fait, peu de temps après la publication de son dernier livre, Keynes a commencé à indiquer son intention d'en produire une nouvelle version (voir Dostaler, 1990).

7. The General Theory is not an easy book to read, in part because the inherently difficult nature of its material does not allow for a simple treatment, and in part because Keynes did not spend (or have) the time necessary to work everything out in a consistent manner. This has given rise to many attempts at exegesis, at finding out what Keynes really meant. This search is complicated by the fact that there are inconsistencies in The General Theory, with some statements or definitions that do not follow from the assumptions on which the analysis is based» (Asimakopulos, 1991, p. xv). 
sitaire, il a consacré très peu de temps à l'étude de la théorie économique. Sa thèse de doctorat était une étude des fondements logiques des probabilités, sur laquelle il a continué de travailler pendant plusieurs années après sa soutenance, avant de la publier finalement en 1921 (CW, vol. 8). Comme Kalecki, il était essentiellement autodidacte en théorie économique. Au cours de son long combat contre Keynes, Hayek a souligné à maintes reprises l'insuffisante maîtrise, par Keynes, des instruments de la théorie économique, les trous parfois étonnants dans sa connaissance de la littérature économique, Keynes ne lisant que ce qui l'intéressait, soit pour des raisons esthétiques, soit parce que les thèses défendues se rapprochaient des siennes. De l'approche intuitive de Keynes, Austin Robinson, membre du fameux Circus de Cambridge qui a secondé Keynes dans le passage du Treatise on Money à la Théorie générale, a donné la surprenante explication suivante: «J'ai depuis longtemps l'impression que la pensée économique de Keynes était en réalité intuitive, impressionniste, et en un sens féminine, plutôt que précise, ordonnée et méticuleuse» (Robinson, E. A. G., 1964, p. 90) ${ }^{8}$. Asimakopulos considère lui aussi que «Keynes, à en juger par ses écrits, n'était pas un théoricien méticuleux» (1991, p. 12). Il estime toutefois que cette lacune est peut-être circonstancielle, liée à la nécessité pour Keynes de publier rapidement ses travaux ${ }^{9}$.

\section{TEMPS, INCERTITUDE ET ÉQUILIBRE}

Mais, plutôt qu'un tempérament «intuitif», c'est peut-être un choix méthodologique qui explique l'approche de Keynes, et une conviction dont on trouve les traces dans ses premiers écrits, à caractère philosophique, de la première décennie du siècle, et dont on entend l'écho dans ce passage du Treatise on Probability: «En tout cas, je n'entretiens pas le même grand espoir que Condorcet, ou même Edgeworth, 'éclairer les Sciences morales et politiques par le flambeau de l'algèbre'» (Keynes, CW, vol. 8, p. 349; le passage entre guillemets est en français dans le texte). Asimakopulos s'attarde peu aux travaux de Keynes antérieurs à la Théorie générale, en particulier à ceux auxquels nous venons de faire allusion, mais nous sommes convaincus qu'ils peuvent aider à éclairer les fondements méthodologiques et philosophiques de ce qu'il appelle la Théorie générale ${ }^{10}$. L'approche de Keynes aux processus sociaux et économiques est une approche en termes de causalité, inscrite dans un temps qu'Asimakopulos qualifie, à la suite de Joan Robinson, d'historique. L'histoire est irréversible. Le passé est révolu. L'analyse économique doit partir d'une situation concrète donnée, dans laquelle

8. Le qualificatif de féminin utilisé par Robinson laisse songeur quand on sait qu'il s'agit de l'époux de Joan Robinson. Jugeait-il que les œuvres de son épouse manquaient d'ordre et de précision, relativement aux siennes?

9. "The rush to get The General Theory into print gave rise to inconsistencies that pose problems of interpretation. There was not enough time for the reflection, and the necessary re-writing, that Keynes needed to ensure that all the elements of his theory were presented in a manner that is consistent with his own final understanding of the outcome of his 'long struggle of escape'». (Asimakopulos, 1991, p. 59).

10. Sans compter que la lecture d'un livre comme le Treatise on Probability demeure très utile pour éclairer certains débats contemporains, outre la lumière qu'elle jette sur la vision philosophique de Keynes, qui détermine son approche à la théorie économique. 
les agents sont contraints par le résultat de décisions prises dans le passé. Il en est ainsi, par exemple, du niveau des dépenses d'investissement. Et l'avenir est incertain. Les choix économiques présents seront suivis d'effets, parfois très lointains, pour lesquels on ne peut établir de probabilités de réalisation. Ces choix ne sont pas et ne peuvent être rationnels ${ }^{11}$. Son article de février 1937 dans le Quarterly Journal of Economics est à cet égard très clair, et Keynes y opère la liaison avec ses préoccupations philosophiques de jeunesse. Il indique que la prise en compte du temps et de l'incertitude constitue l'axe fondamental de son livre et que les méthodes de la théorie classique sont inaptes à expliquer ces phénomènes. C'est sur la même base qu'il critique durement la tentative par Tinbergen de construire un modèle mathématique des fluctuations économiques (CW, vol. 14, 306-20). Pour Keynes, l'approche classique se caractérise par le fait qu'on pense pouvoir prévoir l'avenir avec un certain degré de certitude quantifiable. Les décisions économiques seraient le fruit de la comparaison entre des coûts et des bénéfices futurs qu'on peut évaluer. Tel est le principe de base d'une approche en termes d'équilibre statique à laquelle Keynes s'oppose. Le temps y est de caractère logique plutôt qu'historique, et réversible.

Mais il y a ici un problème important, qu'Asimakopulos souligne à plusieurs reprises, tant dans ses articles que dans son livre, et qui constitue sans doute la principale source des contradictions et des faiblesses de l'œuvre de Keynes. Il s'agit d'une tension continuelle entre l'analyse causale et l'analyse en termes d'équilibre. Car il y a aussi chez Keynes une analyse en termes d'équilibre. On dit ainsi de la Théorie générale que son apport principal est l'explication de la possibilité d'un équilibre de sous-emploi. Asimakopulos explique que le mot équilibre peut être utilisé dans deux sens différents: «L'équilibre peut être défini comme une position de 'repos' pour la variable considérée, ou comme une position 'choisie' par les individus en ce qui concerne leurs actions, compte tenu de la valeur des paramètres" (Asimakopulos, 1991, p. 27) ${ }^{12}$. Dans le premier cas, il caractérise une situation dans laquelle aucune force ne tend à faire se mouvoir un paramètre pour l'amener à un état donné, considéré comme optimal. Telle est la situation pour l'emploi dans une économie de marché, aucune force ne tendant à le pousser spontanément vers le plein emploi. Il s'agit d'un équilibre partiel qui ne s'oppose aucunement à une insatisfaction des agents, et à des mouvements dans les prix et les revenus. Une deuxième conception, souvent confondue avec la première, tend à voir l'équilibre comme une position dans laquelle les agents sont satisfaits de l'état dans lequel ils se trouvent. Les deux visions sont confondues par ceux qui voient dans le modèle

11. Il peut être utile de souligner que Keynes avait entrepris, en 1925, la lecture des œuvres de Freud, et que les remarques éparses qu'on trouve dans ses écrits, à propos des thèses du fondateur de la psychanalyse, ont certainement plus d'importance qu'on ne le croit généralement, y compris pour apprécier certains aspects de l'analyse contenue dans la Théorie générale, par exemple l'allusion aux "esprits animaux» des entrepreneurs comme moteur de l'investissement (voir Winslow, 1986). Deux années après la publication de la Théorie générale, dans son texte, "My Early Beliefs», publié à titre posthume (CW, vol 10, 433-50), Keynes insiste sur l'importance de sa découverte de Freud, qui a contribué à l'aider à couper les derniers ponts avec la «rationalité benthamite» qui constitue le fondement épistémologique des théories classique et néo-classique.

12. Voir aussi Asimakopulos (1989b). 
de Keynes une situation dans laquelle les rigidités de prix et de salaires empêchent les marchés, et en particulier le marché du travail, d'atteindre l'équilibre. Il s'agit là d'une erreur. Keynes croit effectivement que les économies contemporaines se caractérisent par un certain degré de rigidité à la baisse des salaires. Mais une lecture attentive du chapitre 19 de la Théorie générale montre que cette rigidité n'est d'aucune manière une hypothèse essentielle de son modèle, et la cause de l'équilibre de sous-emploi. Ce dont Keynes est par ailleurs convaincu, c'est qu'une politique délibérée de baisse des salaires nominaux ne constitue en aucune manière une garantie de rétablissement du plein emploi.

La Théorie générale peut être ainsi lue autant comme une analyse causale, donnant toute leur importance au temps historique et à l'incertitude, que comme un système d'équilibre général qu'on peut formaliser, par exemple, au moyen du célèbre modèle IS-LM de John Hicks et Alvin Hansen. La situation se complique du fait que le temps de la Théorie générale est celui de la courte période marshallienne. Le glissement est facile en effet de l'analyse de courte période à l'analyse statique, dans laquelle les relations causales entre les variables sont remplacées par des relations fonctionnelles et synchroniques entre des variables interdépendantes $^{13}$. Cette tension explique en grande partie les problèmes dont nous ferons maintenant état.

\section{DEMANDE EFFECTIVE ET FONDEMENTS MICROÉCONOMIQUES MARSHALLIENS}

Dans un article publié en 1982 (1982b), Tom Asimakopulos attire l'attention sur une incohérence logique dans la définition par Keynes de la demande effective ${ }^{14}$. Cette critique s'appuie sur l'interprétation des fondements microéconomiques de l'analyse de Keynes comme marshalliens ${ }^{15}$. Dans un cadre marshallien de concurrence parfaite, les entrepreneurs individuels ne peuvent agir sur les prix, qui sont pour eux des données exogènes. La courbe d'offre globale décrite par Keynes au troisième chapitre de la Théorie générale est une construction théorique dérivée des courbes d'offre individuelles établies selon les mécanismes mis en lumière par Marshall, courbes dans lesquelles les prix constituent les variables indépendantes. Les prix sont remplacés, dans la courbe globale, par les recettes, déduites de l'agrégation des prix et des quantités individuelles. Asimakopulos expose quatre manières différentes d'agréger les courbes individuelles pour construire la courbe globale. Cela ne pose pas de problèmes, à condition de bien voir qu'il ne s'agit pas d'une fonction sur laquelle sont basées les décisions des entrepreneurs. Il n'en est pas de même de la courbe de demande agrégée, définie comme «le 'produit' que les

13. "There is an underlying tension in the exposition of The General Theory between the formal analysis that deals with short-period equilibrium positions, and the specific historical setting of a capitalist economy that is subject to cyclical behaviour-to booms and crises» (Asimakopulos, 1991, p. 83).

14. Il indique que Jack Weldon avait signalé cette erreur en 1975. Casarosa (1981) et Parrinello (1980-81) en ont aussi fait état.

15. C'est dans ce cadre que, par exemple, Keynes déduit l'existence d'une relation décroissante entre le salaire réel et l'emploi. On ne peut toutefois conclure de ce fait en l'existence d'un marché du travail dans lequel serait déterminé l'emploi. 
entrepreneurs espèrent tirer de l'emploi de $N$ personnes» (Keynes [1936] 1982, p. 49) ${ }^{16}$ :

«Il n'y a pas de fondement pour une telle fonction de demande globale 'anticipée' dans son modèle de concurrence. Les prix anticipés des entrepreneurs keynésiens sont indépendants de leur production individuelle (et de l'emploi). La théorie de Keynes utilise par la suite une fonction de demande globale qui est cohérente avec ses fondements microéconomiques. Cette fonction montre la relation entre le niveau d'emploi et le produit qui en résultera. Keynes fait l'hypothèse implicite que ces produits sont ceux qui se réalisent quand l'investissement réalisé est égal à l'investissement planifié et que la consommation est dans une relation désirée avec le revenu.» (Asimakopulos, 1987a, p. 43)

Dans un premier temps, Keynes définit donc erronément la courbe de demande globale comme si les entreprises pouvaient anticiper une action de leur part sur les prix, et cette première définition «ne concorde pas avec les fondements microéconomiques de sa théorie»(1986a, p. 342). Il faut donc définir la demande effective de la manière suivante: "la demande effective est le point situé sur la fonction d'offre agrégée qui correspond aux anticipations de recettes par les entrepreneurs» (Asimakopulos, 1982b, p. 103; italiques dans le texte). Mais cette erreur ne remet pas en question la validité de l'analyse keynésienne de la demande effective, lorsqu'on a bien compris que Keynes fait l'hypothèse que les anticipations des entrepreneurs sont effectivement réalisées ${ }^{17}$. Dans des notes rédigées pour un cours donné en 1937, dans lesquelles il fait allusion aux thèses suédoises, Keynes admet qu'il modifierait désormais sa présentation, en examinant d'abord la détermination de l'emploi dans l'hypothèse où les anticipations sont satisfaites, puis en reprenant l'étude dans le cadre où elles ne le sont pas ${ }^{18}$.

\section{FONCTION DE CONSOMMATION ET MULTIPLICATEUR}

L'analyse de la fonction de consommation et du multiplicateur est un autre lieu de glissement entre une analyse causale se déroulant dans un temps historique et une analyse en termes d'équilibre statique. Il s'y greffe aussi une confusion qu'on retrouve souvent chez Keynes, aussi bien dans les travaux préparatoires à la Théorie générale que dans son livre et dans les articles qui l'ont suivi, entre la perception de l'égalité entre l'épargne et l'investissement comme une identité comptable et comme une condition d'équilibre.

16. Nous avons ici un exemple de la traduction déficiente de la Théorie générale de Keynes. C'est en effet par le mot «produit» qu'est traduit «proceeds» qu'il faudrait rendre par «recettes».

17. "Keynes explains the determination of employment, in any short period, as the result of the interaction of aggregate demand and supply curves. As a pioneer in this work, it is not surprising that he did not succeed in working out a consistent statement of all the basic relations in his model, but his slips do not affect the main conclusions of his analysis. Keynes built on, and adapted, the Marshallian tools of analysis that he took for granted, in order to derive these aggregate relations" (Asimakopulos, 1991, p. 39).

18. "I now feel that if I were writing the book again I should begin by setting forth my theory on the assumption that short-period expectations were always fulfilled; and then have a subsequent chapter showing what difference it makes when short-period expectations are disappointed» (Keynes, CW, vol. 14, p. 181). 
Sur la base des études de Kuznets, faisant état d'une relation stable entre l'épargne et le revenu, Duesenberry, Modigliani et Friedman, parmi d'autres, ont critiqué la relation fonctionnelle établie par Keynes entre la consommation courante et le revenu courant issu de la même courte période. Pour Asimakopulos, la fonction de consommation de Keynes n'implique absolument pas un tel type de rapport. On peut très bien concevoir que le revenu anticipé puisse y figurer comme variable indépendante. Ici encore, Keynes a été plus clair dans des écrits ultérieurs, en particulier dans les notes de cours de 1937 que nous avons déja citées ${ }^{19}$.

La situation est plus délicate dans le cas du multiplicateur, et les circonstances atténuantes moins nombreuses. Le multiplicateur est sensé agir dans la courte période keynéso-marshallienne. Mais dans la plupart de ses présentations, Keynes suppose plutôt une application instantanée du multiplicateur. Nous sommes dans une analyse qui se situe hors du temps, et qui s'adapte bien aux modèles de type IS-LM ${ }^{20}$ :

«Le traitement du multiplicateur par Keynes est confus parce qu'il utilise l'expression de 'propension marginale à consommer' à la fois pour indiquer le résultat de la différenciation de la fonction de consommation, qui montre la relation normale entre les dépenses de consommation et les revenus, et le rapport entre l'accroissement de la consommation et l'accroissement du revenu. [...] Dans tous les cas, on accorde très peu d'attention au temps durant lequel agit le processus du multiplicateur, et l'analyse par Keynes du multiplicateur est essentiellement statique, s'appliquant à la comparaison de deux positions avec des valeurs différentes de l'investissement.» (Asimakopulos 1991, p. 66)

\section{LA DÉTERMINATION DE L'INVESTISSEMENT}

C'est dans l'analyse de la détermination de l'investissement que se manifeste le plus clairement la tension entre l'analyse causale et l'analyse fonctionnellestatique, entre la rupture et la continuité avec la vision classique. Cette question a fait l'objet du premier article important d'Asimakopulos sur la Théorie générale

19. «Keynes's reference to consumption behaviour in the rough notes for his 1937 lectures also shows this increased emphasis on the influence of these expectations. It displays considerable scepticism about the possibiliby of relating consumption to current income. His revised position is compatible with subsequent approaches to this topic, such as the permanent-income hypothesis of Friedman (1957), and the life-cycle hypothesis of Modigliani and Brumberg (1954)» (Asimakopulos, 1991, p. 64). L'opposition radicale entre Friedman et Keynes sur plusieurs plans, en particulier celui des propositions de politique économique, peut ainsi s'accommoder de certaines convergences sur d'autres plans. Ainsi l'un et l'autre sont-ils beaucoup plus proches de Marshall que de Walras et de ses disciples modernes, à qui ils reprochent de préférer l'élégance de modèles mathématiques abstraits au réalisme d'explications ou de prédictions nécessairement incertaines dans le domaine complexe des actions humaines.

20. Hicks a lui-même, à partir milieu des années soixante-dix, commencé à prendre ses distances par rapport à une construction dont il était le principal inspirateur et qui, au bout du compte, évacue l'un des éléments essentiels de l'approche de Keynes: la prise en compte du temps. Mais il faut ajouter que le célèbre «Mr. Keynes and the Classics» (1937) avait été précédé, un an plus tôt, d'une critique tout à fait différente du livre de Keynes, dans laquelle Hicks faisait ressortir l'importance du temps et des anticipations. Hicks a d'ailleurs mentionné l'influence qu'avaient eue sur lui, à cette époque, les écrits des économistes suédois, et en particulier L'Équilibre monétaire de Myrdal (voir à ce sujet Dostaler, 1991). 
de Keynes (Asimakopulos, 1971). La contradiction se manifeste entre deux chapitres consécutifs de la Théorie générale. Dans le premier, le chapitre 11, Keynes élabore le concept d'efficacité marginale du capital dont on peut déduire une courbe à pente négative liant investissement et taux d'intérêt, et qu'on peut considérer comme une courbe de demande d'investissement, autre élément-clé de la synthèse IS-LM. Dans le chapitre suivant, consacré à l'état des prévisions à long terme, Keynes attire l'attention sur le caractère volatil de l'investissement, lié aux anticipations incertaines des entrepreneurs, et dont le niveau dépend de qu'il appelle leurs "esprits animaux». Il n'y a guère de place, dans cette perspective, pour une vision de l'investissement considéré comme la résultante d'un calcul rationnel dans le cadre duquel sont comparés des bénéfices futurs et des coûts présents. Il n'y a en fait guère de place pour une fonction d'investissement. La question est importante. Keynes y revient d'ailleurs dans son article du Quarterly Journal of Economics, insistant sur le fait que le niveau de l'investissement est la causa causans de son modèle, et que sa détermination est aléatoire, volatile, incertaine. De là découle l'instabilité des économies contemporaines, les fluctuations cycliques, la persistance de taux de chômage élevés, phénomènes dont la théorie classique est incapable de rendre compte.

Dans la perspective d'Asimakopulos, c'est la vision du chapitre 11 qui doit céder la place. Comme le multiplicateur, l'efficacité marginale du capital est un concept ambigu. Kalecki avait déjà bien perçu les contradictions de l'analyse keynésienne de la détermination de l'investissement, comme en fait foi la critique qu'il a rédigée, en 1936, du livre de Keynes ${ }^{21}$. Ces contradictions découlent, encore une fois, de problèmes avec le temps. Dans son analyse de l'efficacité marginale du capital, Keynes confond des éléments ex ante et ex post. Deux raisons sont en effet mises de l'avant pour expliquer la diminution de l'efficacité marginale du capital avec l'augmentation de l'investissement. La première tient à la diminution du rendement escompté du capital à mesure qu'augmente sa quantité. La seconde découle de l'augmentation du prix des biens d'investissement. Or, le court terme est défini par Keynes comme une période pendant laquelle le stock de capital est constant, ou sujet à une augmentation si minime qu'on peut en négliger les effets. Dans le cadre temporel d'analyse dans lequel se situe la Théorie générale, la première explication de la baisse de l'efficacité marginale ne peut donc tenir. Keynes se rabat sur l'explication par la hausse du prix des biens d'investissement. Mais il y a ici un autre problème de taille. La courbe décroissante de l'efficacité marginale du capital est obtenue par l'agrégation des courbes individuelles. Or, pour un entrepreneur individuel, la hausse du prix des biens d'investissement n'est pas le résultat de son action, mais bien celle de l'action de l'ensemble des autres entrepreneurs. Et cela ne peut être constaté qu'ex post, et non pas prédit ex ante.

21. Dans l'introduction, publiée à titre posthume, de ce qu'il considère être ses principales contributions à l'étude de la dynamique des économies capitalistes contemporaines, Kalecki indique alors que la théorie de la demande effective se trouve clairement énoncée dans ses premiers travaux - et évidemment dans la Théorie générale de Keynes, que les dits travaux avaient donc anticipée - l'analyse de la détermination du niveau de l'investissement constitue un «talon d'Achille» de la théorie économique, problème auquel il a cherché jusqu'à la fin de sa vie à apporter une solution satisfaisante (Kalecki, 1971). 
Nous avons ici, de nouveau, un problème avec le traitement du temps. Et, comme pour la courbe de demande agrégée, se pose ici encore un problème de passage des fondements microéconomiques aux concepts globaux:

«Des concepts ex ante et ex post sont impliqués ici; les rendements escomptés qui sont à la base de l'efficacité marginale du capital sont clairement ex ante dans cette formulation, mais les prix d'offre croissants sont en partie ex post [...]

Non seulement l'efficacité marginale du capital est-elle basée sur ce mélange de facteurs ex ante et ex post, mais elle est sélective dans ce qu'elle inclut des derniers, de manière à obtenir une courbe décroissante unique. Les rendements escomptés dépendent en grande partie de la quantité de capital existant, de son degré d'utilisation, de l'état de la technologie, ainsi que des taux récents de prix et de salaires. Ils sont donc affectés par le niveau actuel de l'investissement, mais on ne trouve aucune allusion à ce fait dans le traitement habituel de la fonction de demande d'investissement de Keynes. On inclut l'impact d'un accroissement du taux d'investissement sur les prix des biens capitaux, mais pas son effet sur les profits espérés. Ce dernier facteur doit aussi être introduit pour obtenir un tableau plus clair des multiples éléments du modèle de Keynes et de leur interaction.» (Asimakopulos, 1971, p. 383-4)

Acceptant ce qu'il appelle le premier postulat de l'école classique, liant le salaire réel au produit marginal du travail, Keynes aurait dû ainsi déduire que l'augmentation de l'investissement, en accroissant les profits, augmente le rendement escompté du capital, mais il ne tient aucun compte de cet effet. Pour corriger la formulation déficiente de Keynes, il faut revenir à la vision élaborée par Kalecki dans son travail de 1933, établissant une double relation entre le taux de profit et l'investissement. Les conditions courantes jouent un rôle dans les anticipations, et le taux de rendement escompté de l'investissement est ainsi lié, entre autres, au niveau actuel de l'investissement et au niveau des profits qui lui est associé. En s'inspirant de l'illustration proposée par Joan Robinson du rapport entre le taux de profit et le taux d'accumulation, Asimakopulos représente cette première relation par une courbe $\mathrm{E}$, qui exprime le lien entre le niveau actuel de l'investissement et le taux de profit escompté. Une autre fonction, illustrée par une courbe I, exprime le lien entre le taux de rendement escompté et le niveau d'investissement qu'il suscite (1971, p. 386). Cette fonction, qui dépend tant de facteurs non économiques qu'économiques, inclut en particulier les effets des "esprits animaux" des entrepreneurs. Rien ne garantit que ces deux courbes se croisent à un niveau d'investissement qui soit suffisant pour établir le plein emploi (1982, p. 387). En s'inspirant toujours de l'analyse de Kalecki, on peut compliquer cette double relation en introduisant les délais qui interviennent entre la décision d'investir, la production des biens d'investissement et leur livraison. Cette construction, plus complexe que la courbe d'efficacité marginale du capital qu'on trouve à la base du modèle IS-LM, illustre mieux les relations causales à l'œuvre dans la Théorie générale. Elle n'en modifie pas les conclusions. Le message central de Keynes demeure toujours le même, et il est mieux étayé. Aucune force n'assure l'établissement d'un équilibre de plein emploi au niveau agrégé et l'investissement est une activité dont l'extrême 
volatilité est en définitive responsable des fluctuations cycliques de l'activité économique et de l'équilibre de sous-emploi ${ }^{22}$.

\section{MONNAIE ET FINANCEMENT}

Nonobstant de nombreuses différences, parfois substantielles, tant dans les problèmes considérés que dans les méthodes d'analyse mises en œuvre, il y a un élément commun essentiel à l'analyse de Keynes et à celle de Kalecki, comme de leurs disciples post-keynésiens. L'investissement y est conçu comme une donnée exogène, déterminée dans la courte période par des décisions prises dans le passé, et, surtout, indépendante de l'épargne. Comme le dit Joan Robinson, la caractéristique des théories post-keynésiennes de la croissance, par opposition aux théories néo-classiques, est que les entreprises sont libres d'acccumuler comme elles le désirent. Elles ne sont pas contraintes par un fonds d'épargne préalable.

Cette idée est déjà présente dans le Treatise on Money de Keynes, et elle constitue à notre avis le principal point en litige dans le débat qui se déroule alors entre Keynes et Hayek. Ce débat plonge ses racines dans un passé lointain, bien antérieur à Adam Smith et il continue à occuper le devant de la scène dans les controverses actuelles en macroéconomie. Dans son plus récent ouvrage, Hayek en a même décelé des fondements éthiques, attribuant la vision de Keynes à son immoralisme (Dostaler, 1990).

Dans la Théorie générale, l'investissement génère l'épargne, qui lui est égale ex post, au moyen de variations dans le niveau de la production. L'effet du multiplicateur assure que le montant de l'épargne sera dans la relation souhaitée avec le revenu. Keynes n'aborde pas, dans son livre, la question du financement de l'investissement. Kalecki avait déjà attiré l'attention, dans un article publié en 1935, sur la nécessité d'une inflation de crédit pour permettre le financement de l'investissement. L'investissement ne peut s'accroître que si le système bancaire injecte les liquidités nécessaires à son financement. Les effets de l'investissement permettront ensuite de générer les fonds qui permettront aux entreprises de rembourser leurs dettes. La monnaie est ainsi créée ex nihilo, pour répondre aux besoins de financement des entreprises, et elle constitue un fonds de roulement qui retourne à la fin du circuit à son point de départ. On trouve aussi chez Schumpeter, et avant lui chez Wicksell, cette vision d'une monnaie créée de manière endogène au système. Elle s'oppose évidemment à la conception exogène de l'offre de monnaie qu'on trouve dans la Théorie générale. Mais, dans le débat sur le taux d'intérêt qui l'oppose à Ohlin dans l'année qui suit la publication de son livre, Keynes se rallie à la position de Kalecki, et ajoute aux éléments déterminants la demande de monnaie le financement de l'investissement ${ }^{23}$.

22. "The distinct possibility of unemployment equilibrium, because of the unreliability and volatility of investment is the 'central message' of the General Theory" (Asimakopulos, 1991, p. 102).

23. "They [trois articles postérieurs à la Théorie générale, GD] contain a minor amendment to his model, the recognition of the increased demand for money when investment decisions are increased. The banking system is assumed to accommodate this demand-he calls it a demand for 'finance'-by increasing the money supply, and thus the demand for money is given a role in determining this supply" (Asimakopulos 1991, p. 109-10). 
Cette perspective a été développée par les post-keynésiens américains (Davidson, Minsky) et par les théoriciens du circuit en France (Parguez, Poulon, Schmitt). Asimakopulos y décèle quelques failles sérieuses, liées encore une fois à un traitement déficient du temps. En effet, Keynes ne parvient pas vraiment à établir la totale indépendance de l'investissement par rapport à l'épargne, dans toutes les circonstances, à moins de supposer un fonctionnement instantané du multiplicateur. De son analyse de la question du financement des investissements, Tom Asimakopulos conclut que l'épargne peut jouer un certain rôle causal dans le processus de détermination de l'investissement ${ }^{24}$.

\section{CROISSANCE ET ÉQUILIBRE A LONG TERME}

Un des axes essentiels de la réflexion post-keynésienne a consisté à dynamiser la Théorie générale en en généralisant les conclusions pour l'étude de la croissance. Roy Harrod et Joan Robinson sont deux des principaux artisans de cet effort théorique. De plus en plus critique, au fil des ans, face à leurs analyses, Tom Asimakopulos en constate l'échec dans son dernier livre. Cet échec est lié, encore une fois, à la tension entre l'analyse causale et l'analyse en termes d'équilibre. Les sentiers de croissance de diverses natures proposés par les économistes de Cambridge supposent tous un équilibre de longue période qui est incompatible avec l'approche de Keynes. Il n'y a pas et ne peut y avoir, dans la Théorie générale de niveau d'équilibre de plein emploi en longue période ${ }^{25}$.

Reprenant une formulation de Kalecki, Asimakopulos indique que la longue période n'est qu'une succession de courtes périodes, au cours desquelles les anticipations des agents peuvent constamment se révéler déçues. Asimakopulos oppose ainsi sa vision de la longue période à celle de Joan Robinson, en lui reprochant d'oublier qu'il s'agit finalement d'un intervalle de temps, pendant lequel l'investissement fait croître le stock de capital, et non pas d'une certaine forme d'état d'équilibre et de tranquillité.

C'est le même type de critique qu'Asimakopulos oppose à ceux qui cherchent à effectuer une synthèse entre l'approche keynésienne et celle développée par Sraffa dans Production de marchandises par des marchandises (1960). L'objectif de Sraffa est tout à fait différent de celui de Keynes. Il s'agit en effet pour lui d'indiquer

24. "None of these criticisms affect the conclusions of Kalecki and Keynes on the critical role of the rate of investment in determining effective demand, on the possibility of equilibrium with involuntary unemployment and on the possibly large fluctuations in the rate of investment because of changing views concerning a future about which there is a great deal of uncertainty. What they do is to indicate that there may be limits, related in some way to the propensity to save, to the extent to which firms are in a position to increase their rate of investment even if short-term credit is available to finance such an increase» (Asimakopulos [1983b] 1988a, p. 163-4). Cet article a déclenché une vive polémique. Voir en particulier Davidson (1986), Graziani (1984, 1985) et Kregel (1986). Voir aussi Asimakopulos (1985b, $1985 \mathrm{c}, 1986 \mathrm{~b}, 1986 \mathrm{c}$ et $1986 \mathrm{~d})$.

25. "Long-period equilibrium has no place in Keynes's analytical framework since the stationary conditions that are required to make long-period equilibrium values centres of attraction for actual values are at variance with Keynes's vision of capitalist economies that are subject to changes from many sources» (Asimakopulos 1991, p. 26). Voir aussi Asimakopulos (1984-85). 
comment sont déterminés les prix et la répartition des revenus dans une économie capitaliste moderne. Pour ce faire, Sraffa développe une analyse d'équilibre statique dont la teneur est en fait contradictoire avec l'approche méthodologique préconisée par Keynes. Ce que d'aucuns ont appelé la "synthèse post-classique», unissant les apports des courants post-keynésiens et néo-ricardiens, serait donc impossible:

«Keynes était préoccupé par ces problèmes pratiques et l'emphase placée sur la courte période est une force de son analyse, alors que Sraffa se préoccupait de questions théoriques hautement abstraites; dans cette optique, une analyse préoccupée seulement d'équilibre de longue période n'est pas inappropriée. Mais dans ces travaux il n'y a pas de point commun entre Sraffa et Keynes.» (Asimakopulos 1982a, p. 93) ${ }^{26}$

\section{CONCLUSION}

Dans son dernier livre, comme dans l'ensemble de ses articles sur Keynes, Tom Asimakopulos met de l'avant une interprétation originale, susceptible d'alimenter et d'éclairer les débats actuels en macroéconomie. La Théorie générale de Keynes, œuvre fondatrice de la macroéconomie moderne, demeure toujours au centre de ces débats, qu'on admette ou qu'on rejette la problématique de Keynes, car «les questions qu'il a soulevées, comme les problèmes du chômage involontaire, la volatilité de l'investissement, et la complexité des arrangements monétaires dans une économie capitaliste moderne, sont toujours avec nous» (Asimakopulos 1991, p. Xv).

Keynes nous a laissé une œuvre tout à la fois riche, complexe et contradictoire. La Théorie générale n'en constitue qu'un élément. Elle est l'aboutissement d'un long processus d'élaboration dont la genèse se trouve dans les réflexions sur l'éthique et les fondements logiques des probabilités que Keynes menait dans les premières années de notre siècle, comme dans la vision politique qui était déjà la sienne. On reprochera, peut-être, à Tom Asimakopulos de ne pas tenir suffisamment compte de ses développements préalables, de l'ensemble de l'œuvre de Keynes. Mais en même temps qu'elle constitue un aboutissement, la Théorie générale est le point de départ de nouveaux développements dans lesquels Keynes lui-même, mais aussi d'autres acteurs sont aussi intervenus. Ce sont ces nouveaux développements qui intéressent Asimakopulos. Il s'agit, à partir d'une Théorie générale incomplète et porteuse de contradictions, de construire une Théorie générale susceptible d'éclairer les problèmes de notre temps. Dans cette tâche, Tom Asimakopulos nous a laissé, comme testament théorique, une contribution importante, résultat d'une longue fréquentation de l'œuvre de Keynes, d'efforts incessants pour en expliquer le sens, mais aussi d'une intense réflexion sur la nature et le fonctionnement des économies contemporaines. 


\section{BIBLIOGRAPHIE}

ASIMAKOPULOS, A. 1971. «The Determination of Investment in Keynes's Model», Revue canadienne d'économique, vol. 4, 382-88.

Asimakopulos, A. 1973. «Keynes, Patinkin, Historical Time, and Equilibrium Analysis", Revue canadienne d'économique, vol. 6, 179-88.

AsIMAKOPULOS, A. 1978. «Keynesian Economics, Equilibrium, and Time», Revue canadienne d'économique, vol. 11, supplément, S3-S10.

AsImAKopulos, A. 1982a. «Keynes et Sraffa», L'Actualité économique, vol. 58, 87-94.

Asimakopulos, A. 1982b. «Keynes' Theory of Effective Demand Revisited», Australian Economic Papers, vol. 21,18-36; in AsIMAKOPULOS (1988a), 100-26.

ASIMAKOPULOS, A. 1983a. "Anticipations of Keyne's General Theory?», Revue canadienne d'économique, vol. 16, 517-30; in ASIMAKOPULOS (1988a), 167-85.

AsIMAKopulos, A. 1983b. «Kalecki and Keynes on Finance, Investment and Saving", Cambridge Journal of Economics, vol. 7, 221-34; in ASIMAKOPULOS (1988a), 147-66.

Asimakopulos. A. 1984. "The General Theory and Its Marshallian Microfundations», Metroeconomica, vol. 36, 161-77.

ASIMAKOPULOS, A. 1984-85. "'Long-period Employment' in The General Theory", Journal of Post Keynesian Economics, vol. 7, 207-13.

Asimakopulos, A. 1985a. «Keynes and Sraffa: Visions and Perspective», Political Economy: Studies in the Surplus Approach, vol. 1, 33-50; in ASIMAKOPULOS (1988a), 127-46.

AsimaKopulos, A. 1985b. "Finance, Saving and Investment in Keynes's Economics: A Comment», Cambridge Journal of Economics, vol. 9, 405-7.

ASIMAKOPULOS, A. 1985c. "The Role of Finance in Keynes's General Theory", Economic Notes, vol. 14, 5-17.

ASIMAKOPULOS, A. 1986a. «Les théories keynésiennes d'accumulation et leur incidence sur l'élaboration des politiques: un examen critique", in vol. 21 des études commandées par la Commission royale sur l'union économique et les perspectives de développement du Canada, sous la direction de JOHN SARGENT, Ottawa, ministre des Approvisionnements et Services, 337-86.

ASIMAKOPULOS, A. 1986b. «Finance, Investment and Saving: A Reply to Terzi», Cambridge Journal of Economics, vol. 10, 81-2.

ASIMAKOPULOS, A. 1986c. «Richardson on Asimakopulos on Finance: A Reply», Cambridge Journal of Economics, vol. 10, 199-201.

ASIMAKOPULOS, A. 1986d. «Finance, Saving and Investment», Journal of PostKeynesian Economics, vol. 9, 79-90. 
ASIMAKOPULOS, A. 1987a. «La signification théorique de la Théorie générale de Keynes», in La "Théorie générale» et le keynésianisme, sous la direction de G. BOISMENU et G. Dostaler, Montréal, ACFAS, 39-54.

ASIMAKOPULOS, A. 1987b. "Approach to Macroeconomics: Keynes and Others», in Keynesian Theory, Planning Models and Quantitative Economics: Essays in Memory of Vittorio Marrama, sous la direction de G. GANDOLFO et F. Marzano, Milan, Giuffre, vol. 1, 3-21.

ASIMAKOPUlOS, A. 1988a. Investment, Employment and Income Distribution, Cambridge, Polity Press; Boulder, Colorado, Westview Press.

ASIMAKOPULOS, A. 1988b. "The Aggregate Supply Function and the Share Economy: Some Early Drafts of the General Theory", in Keynes and Public Policy after Fifty Years, sous la direction de O. HAMOUDA et J. N. SMITHIN, Aldershot, Hants, Edward Elgar, 70-80.

ASIMAKOPULOS, A. 1988c. «The Theoretical Significance of Keynes's General Theory», Thames Papers in Political Economy, été.

ASIMAKOPULOS, A. 1988d. "Kalecki and Robinson: an 'Outsider's Influence'», Journal of Post-Keynesian Economics, vol. 11, 261-78.

ASIMAKOPUlOS, A. 1989a. "Keynes and Sraffa: Visions and Perspectives», in Essays on Piero Sraffa, sous la direction de K. BHARADWAJ et B. SCHEFOLD, Londres, Unwin Hyman, 331-45.

ASIMAKOPULOS, A. 1989b. «The Nature and Role of Equilibrium in Keynes's General Theory», Australian Economic Papers, vol. 28, 16-28.

ASIMAKOPULOS, A. 1989c. "Kalecki and Robinson», in Kalecki's Relevance Today, sous la direction de M. SEBASTIANI, Londres, Macmillan, 10-24.

ASIMAKOPULOS, A. 1990a. "Kalecki and Keynes: Their Correspondence», History of Political Economy, vol. 22, 49-63.

Asimakopulos, A. 1990b. «The Theoretical Significance of Keynes's General Theory", in Theory and Policy in Political Economy: Essays in Pricing, Distribution and Growth, sous la direction de P. ARESTIS et Y. KITROMILIDES, Aldershot, Hants, Edward Elgar.

AsimaKopulos, A. 1991. Keynes's General Theory and Accumulation, Cambridge, Cambridge University Press.

CASAROSA, C. 1981. "The Microfoundations of Keynes's Aggregate Supply and Aggregate Demand Analysis», Economic Journal, vol. 91, 188-93.

DAVIDSON, P. 1986. «Finance, Funding, Savings and Investment», Journal of Post-Keynesian Economics, vol. 9, 101-10.

DostaleR, G. 1988. «La théorie post-keynésienne, la Théorie générale et Kalecki», Cahiers d'économie politique, n 14-15, 123-42.

DOSTALER, G. 1990. «Aperçus sur la controverse entre Keynes et Hayek», Économies et sociétés, vol. $24, \mathrm{n}^{0} 6,135-62$. 
DoSTALER, G. 1991. «Les premiers travaux économiques de Gunnar Myrdal», L'Actualité économique, vol. 67, 192-217.

GrAZIANI, A. 1984. «The Debate on Keynes's Finance Motive», Economic Notes, vol. 31, 5-32.

GrAZIANI, A. 1985. «Le débat sur le 'motif de financement' de J. M. Keynes», Économie appliquée, vol. $38, \mathrm{n}^{\circ} 1,159-75$.

HAMOUDA, Omar 1990. "Athanasios (Tom) Asimakopulos, (1930-1990)», L'Actualité économique, vol. 66, 365-67.

HARCOURT, G.C. 1991. «Athanasios (Tom) Asimakopulos, 1930-1990: A Memoir», Journal of Post Keynesian Economics, vol. 14, 39-48.

HICKS, J. R. 1936. «Mr. Keynes's Theory of Employment», Economic Journal, vol. $46,238-53$.

HICKS, J. R. 1937. «Mr. Keynes and the 'Classics'; A Suggested Interpretation», Econometrica, vol. 5, 147-59.

KAlecki, M. 1933. Proba teorii koniunktury, [Essais sur la théorie du cycle économique] Varsovie, Instytut Badania Koniunkture Gospodarczych i Cen.; trad. anglaise partielle in KALECKI (1971), 1-14.

KALECKI, M. 1935. "A Macrodynamic Theory of the Business Cycles», Econometrica, vol. 3, 326-44.

KALECKI, M. 1936. «Pare uwag o teorii Keynesa», [Quelques remarques sur la théorie de Keynes], Ekonomista, vol. 36, 18-36; in F. TARGETTI et B. KINDAHASS, «Kalecki's Review of Keynes' General Theory» Australian Economic Papers, vol. 21, 244-60.

KALECKI, M. 1942 »A Theory of Profits», Economic Journal, vol. 52, 258-67.

KALECKI, M. 1971. Selected Essays on the Dynamics of the Capitalist Economy, 1933-1970, Cambridge, Cambridge University Press.

KEYNES, J. N. 1936. The General Theory of Employment, Interest and Money, Londres, Macmillan; CW, VII; trad. franç., Théorie générale de l'emploi, de l'intérêt et de la monnaie, Paris, Payot, 1942; éd. de poche, 1982.

KEYNES, J. N. 1971-1989. The Collected Writings of John Maynard Keynes, 30 volumes, Londres, Macmillan.

KREGEL, J. 1986. "A Note on Finance», Journal of Post-Keynesian Economics, vol. 9, 91-100.

PARRINello, S. 1980-81. "The Price Level Implicit in Keynes' Effective Demand", Journal of Post Keynesian Economics, vol. 3, 63-78.

Patinkin, D. 1982. Anticipations of the General Theory? And Other Essays on Keynes, Chicago, University of Chicago Press.

RoBINSON, E. A. G. 1964. «Could There Have Been a 'General Theory' Without Keynes", in Keynes' General Theory: Reports of Three Decades, sous la direction de R. LEKACHMAN, 87-95. 
ROBINSON, J. 1948. «La théorie générale de l'emploi», Économie appliquée, vol. $1,185-96$.

Sraffa, P. 1960. Production of Commodities by Means of Commodities, Cambridge, Cambridge University Press.

WINSLOW, E. G. 1986. «Keynes and Freud: Psychoanalysis and Keynes's Account of the 'Animal Spirits' of Capitalism», Social Research, vol. 53, 549-78. 